\title{
Due-Window Assignment Scheduling with Variable Job Processing Times
}

\author{
Yu-Bin $\mathrm{Wu}^{1}$ and Ping $\mathrm{Ji}^{2}$ \\ ${ }^{1}$ School of Science, Shenyang Aerospace University, Shenyang 110136, China \\ ${ }^{2}$ Department of Industrial and Systems Engineering, The Hong Kong Polytechnic University, Hung Hom, Kowloon, Hong Kong \\ Correspondence should be addressed to Yu-Bin Wu; wuyubin79@163.com
}

Received 18 August 2014; Accepted 11 September 2014

Academic Editor: Dehua Xu

Copyright (c) 2015 Y.-B. Wu and P. Ji. This is an open access article distributed under the Creative Commons Attribution License, which permits unrestricted use, distribution, and reproduction in any medium, provided the original work is properly cited.

\begin{abstract}
We consider a common due-window assignment scheduling problem jobs with variable job processing times on a single machine, where the processing time of a job is a function of its position in a sequence (i.e., learning effect) or its starting time (i.e., deteriorating effect). The problem is to determine the optimal due-windows, and the processing sequence simultaneously to minimize a cost function includes earliness, tardiness, the window location, window size, and weighted number of tardy jobs. We prove that the problem can be solved in polynomial time.
\end{abstract}

\section{Introduction}

In most scheduling studies, job processing times are treated as constant numbers; however, in many practical situations, job processing times are affected by the learning effects and/or deteriorating (aging) effects. Learning effects and deteriorating (aging) effects are important for production and scheduling problems. For details on this line of the scheduling problems with learning effects (deteriorating effects), the reader is referred to a comprehensive survey by Biskup [1] (Gawiejnowicz [2]). Rudek [3] considered single machine scheduling problems with position-dependent job processing times (i.e., learning and aging effects). For the following objectives, the makespan with release dates, the maximum lateness, and the number of late jobs, they gave some results. J.-B. Wang and M.-Z. Wang [4] and Sun et al. [5] considered flow shop scheduling problems with general positiondependent learning effects. For some regular objective functions, they proposed heuristics. Sun et al. [6] considered flow shop scheduling problems with three special positiondependent learning effects. For the total weighted completion time minimization problem, they proposed heuristics. Lu et al. [7] considered single machine scheduling problems with learning effects and controllable processing times. For two due date assignment methods, they presented a polynomialtime optimization algorithm to minimize a multiobjective cost function.

J.-B. Wang and M.-Z. Wang [8] considered common due-window single machine scheduling with learning effects and controllable processing times. For a mule-objective cost function, they presented a polynomial-time optimization algorithm. J.-B. Wang and M.-Z. Wang [9] considered single machine scheduling problems with nonlinear deterioration. They showed that the makespan minimization problem can be solved in polynomial time. J.-B. Wang and M.-Z. Wang [10] considered three-machine flow shop scheduling with deteriorating jobs. For the makespan minimization problem, they proposed a branch-and-bound algorithm and two heuristic algorithms. X.-R Wang and J.-J. Wang [11] considered single machine scheduling problems with deteriorating jobs and convex resource dependent processing times. Xu et al. [12] considered single machine group scheduling with proportional linear deterioration and ready times. For the makespan minimization problem, they gave some results. Cheng et al. [13] considered a single machine common due-window assignment scheduling problem with deteriorating jobs. For a deteriorating maintenance activity, they provided polynomial-time solutions for a multiobjective 
cost. Yang et al. [14] considered a single machine multiple common due dates assignment resource allocation scheduling problems with general position-dependent deterioration effect. For a multiobjective cost, they proved that the problems can be solved in polynomial time, respectively. Liu et al. [15] considered single-machine common due-window assignment scheduling problem with deteriorating jobs. If the width of the common due-window is a given constant, they proved a mule-objective function cost problem can be solved in polynomial time. J.-B. Wang and C. Wang [16] and Wang et al. [17] considered due-window assignment scheduling problems with learning effects and deteriorating jobs at the same time.

The recent paper $\mathrm{Li}$ et al. [18] addresses single machine scheduling problem with deteriorating jobs. For common due date assignment $(\mathrm{CON})$ and common flow allowance (i.e., all jobs have slack due date (SLK)) due date assignment methods, they showed that a multiobjective minimization problem can be solved in polynomial time, respectively. In this research, we continue the work of Li et al. [18] but focus on the common due-window assignment (CONW) scheduling problem (Yin et al. [19]). Under the learning effect and deteriorating jobs models, we prove that the CONW due-window assignment scheduling is solvable in polynomial time, respectively.

\section{Problem Formulation}

The following notations will be used throughout the paper:

$J_{j}$ : Job $j$

$J$ : Set of jobs (i.e., $J=\left\{J_{1}, J_{2}, \ldots, J_{n}\right\}$ )

$C_{j}$ : Completion time of job $J_{j}$

$d_{1}$ : Earliest due date

$D$ : Common due-window size

$d_{2}$ : Latest due date $=d_{1}+D$

$E_{j}$ : Earliness of $J_{j}=\max \left\{0, d_{1}-C_{j}\right\}$

$T_{j}$ : Tardiness of $J_{j}=\max \left\{0, C_{j}-d_{2}\right\}$

$E$ : Set of earliest jobs $=\left\{J_{j} \mid C_{j}<d_{1}\right\}$

$T$ : Set of tardy jobs $=\left\{J_{j} \mid C_{j}>d_{2}\right\}$

$\bar{D}$ : Set of on time jobs (i.e., $\bar{D}=J \backslash(E \cup T))$

$m$ : Number of set $\bar{D}$ jobs (i.e., $m=|\bar{D}|$ )

$\gamma_{j}$ : The penalty weight if $J_{j}$ is tardy (i.e., $J_{j} \in T$ )

$F\left(d_{1}, D, \pi\right)=\alpha d_{1}+\beta D+\theta \sum_{J_{j} \in E} E_{j}+\sum_{J_{j} \in T} \gamma_{j}$ : The total cost function, where $\alpha>0, \beta>0$, and $\theta>0$ are the unit due-window starting time, due-window size, and earliness penalties, respectively.

Consider a nonpreemptive single machine setting. There are $n$ independent jobs $J=\left\{J_{1}, J_{2}, \ldots, J_{n}\right\}$ available at zero and preemption is not allowed. Let $P_{j}$ denote the actual processing time for job $J_{j}$. In this research, we consider the following models.

Job Time-Dependent Deterioration Effect Model (See Li et al. [18]). Consider

$$
P_{j}=a_{j}+b t
$$

where $a_{j}, b>0, t$ are the basic (normal) processing time of $J_{j}$, the deteriorating rate, and the starting time of $J_{j}$, respectively.

Job-Position-Dependent Learning Effect Model (See Biskup [20]). Consider

$$
P_{j}=a_{j} r^{a}
$$

where $a_{j}, a<0, r$ are the basic (normal) processing time of $J_{j}$, the learning rate, and the position $J_{j}$ in a processing sequence, respectively.

Our task of this paper is to determine the optimal earliest due date $d_{1}$, the common due-window size $D$, and a schedule $\pi$ which minimizes the following objective function:

$$
F\left(d_{1}, D, \pi\right)=\alpha d_{1}+\beta D+\theta \sum_{J_{j} \in E} E_{j}+\sum_{J_{j} \in T} \gamma_{j} .
$$

Then, using the common three-field notation introduced by Graham et al. [21], the corresponding scheduling problems are denoted by

$$
\begin{gathered}
1\left|P_{j}=a_{j}+b t\right| \alpha d_{1}+\beta D+\theta \sum_{J_{j} \in E} E_{j}+\sum_{J_{j} \in T} \gamma_{j}, \\
1\left|P_{j}=a_{j} r^{a}\right| \alpha d_{1}+\beta D+\theta \sum_{J_{j} \in E} E_{j}+\sum_{J_{j} \in T} \gamma_{j} .
\end{gathered}
$$

\section{Optimal Solutions}

\subsection{Job Time-Dependent Deterioration Effect Model}

Lemma 1 (Li et al. [18]). For a given schedule $\pi=\left(J_{[1]}, J_{[2]}\right.$, $\left.\ldots, J_{[n]}\right)$, if the starting time of the first job is 0 , then $C_{[r]}=$ $\sum_{j=1}^{r} a_{[j]}(1+b)^{r-j}$ and $\sum_{j=1}^{n} C_{j}=\sum_{j=1}^{n} a_{[j]} \sum_{i=0}^{n-j}(1+b)^{i}$.

Lemma 2. If $\alpha>\beta$, an optimal schedule exists in which the due-window starts at time zero.

Proof. Suppose $\alpha>\beta$, and $d_{1}>0$; we shift $X$ units of time to the left. The change in the total cost is given by $\Delta Z=$ $-\alpha X+\beta X-\theta l X$, where $l$ denotes the number of early jobs. Cleary, $\Delta Z<0$. Therefore, a shift of $d_{1}$ (until $d_{1}=0$ ) can only decrease the total cost.

Lemma 3. An optimal schedule exists in which the duewindow starting time (i.e., $d_{1}$ ), and the due-window completion time (i.e., $d_{2}$ ) coincide with job completion times, respectively.

Proof. Suppose that there exists a schedule starting at time zero and containing jobs at the $k$ th and the $(k+m)$ th positions such that $C_{k}<d_{1}<C_{k+1}, C_{k+m}<d_{2}<C_{k+m+1}$. 
When we shift $d_{2}$ to $C_{k+m}$, the change in the total cost is given by $-\beta\left(d_{2}-C_{k+m}\right)$.

When we shift $d_{1}$ to $C_{k}$, the change in the total cost is given by $(-\alpha+\beta+k \theta)\left(d_{1}-C_{k}\right)$.

When we shift $d_{1}$ to $C_{k+1}$, the change in the total cost is given by $-(-\alpha+\beta+k \theta)\left(C_{k+1}-d_{1}\right)$.

Again, a shift of $d_{1}$ to $C_{k}$ or to $C_{k+1}$ does not increase the total cost.

Therefore, an optimal schedule exists such that both $d_{1}$ and $d_{2}$ coincide with job completion times.

Lemma 4. An optimal schedule exists in which the index of the job completed at the due-window starting time is $k=\lceil(\beta-$ $\alpha) / \theta\rceil$.

Proof. Using the classical small perturbation technique (see J.-B. Wang and C. Wang [16] and J.-B. Wang and M.-Z. Wang [8]), we measure the change in the total cost when moving $d_{1}$.

We shift $d_{1}, X$ units of time to the left, and the effect of the total cost is

$$
-\alpha X+\beta X-\theta(k-1) X
$$

We shift $d_{1}, X$ units of time to the right, and the effect of the total cost is

$$
\alpha X-\beta X+\theta k X
$$

Both expressions (5) and (6) are clearly nonnegative due to the optimality of the original solution.

From $-\alpha X+\beta X-\theta(k-1) X \geq 0$ and $\alpha X-\beta X+\theta k X \geq 0$ we have $k \leq((\beta-\alpha) / \theta)+1$ and $k \geq(\beta-\alpha) / \theta$. And from the integrality of $k$, it follows that $k=\lceil(\beta-\alpha) / \theta\rceil$.

Lemma 5. For the problem $1\left|P_{j}=a_{j}+b t\right| \alpha d_{1}+$ $\beta D+\theta \sum_{J_{j} \in E} E_{j}+\sum_{J_{j} \in T} \gamma_{j}$, if the job sequence is $\pi=$ $\left(J_{[1]}, J_{[2]}, \ldots, J_{[n]}\right)$ and $m=|\bar{D}|$, then the objective function can be expressed as

$$
F\left(d_{1}, D, \pi, m\right)=\sum_{j=1}^{k+m} w_{j} a_{[j]}+\sum_{j=k+m+1}^{n} \gamma_{[j]},
$$

where

$$
w_{j}= \begin{cases}\left\{(\alpha+k \theta)+\beta\left[(1+b)^{m}-1\right]\right\}(1+b)^{k-j} \\ -\theta \sum_{i=0}^{k-j}(1+b)^{i}, & j=1,2, \ldots, k \\ \beta(1+b)^{k+m-j}, & j=k+1, k+2, \ldots, k+m .\end{cases}
$$

Proof. By Lemmas 1 and 3, we have

$$
d_{1}=C_{[k]}=\sum_{j=1}^{k} a_{[j]}(1+b)^{k-j}
$$

$$
\begin{aligned}
& D=C_{[k+m]}-C_{[k]} \\
& =\sum_{j=1}^{k+m} a_{[j]}(1+b)^{k+m-j}-\sum_{j=1}^{k} a_{[j]}(1+b)^{k-j} \\
& =\sum_{j=1}^{k} a_{[j]}(1+b)^{k+m-j}+\sum_{j=k+1}^{k+m} a_{[j]}(1+b)^{k+m-j} \\
& -\sum_{j=1}^{k} a_{[j]}(1+b)^{k-j} \\
& =\sum_{j=1}^{k} a_{[j]}(1+b)^{k-j}(1+b)^{m}+\sum_{j=k+1}^{k+m} a_{[j]}(1+b)^{k+m-j} \\
& -\sum_{j=1}^{k} a_{[j]}(1+b)^{k-j} \\
& =\sum_{j=1}^{k} a_{[j]}(1+b)^{k-j}\left[(1+b)^{m}-1\right] \\
& +\sum_{j=k+1}^{k+m} a_{[j]}(1+b)^{k+m-j}, \\
& F\left(d_{1}, D, \pi, m\right) \\
& =\alpha d_{1}+\beta D+\theta \sum_{J_{j} \in E} E_{j}+\sum_{J_{j} \in T} \gamma_{j} \\
& =\alpha C_{[k]}+\beta\left\{\sum_{j=1}^{k} a_{[j]}(1+b)^{k-j}\left[(1+b)^{m}-1\right]\right. \\
& \left.+\sum_{j=k+1}^{k+m} a_{[j]}(1+b)^{k+m-j}\right\} \\
& +\theta \sum_{j=1}^{k}\left(C_{[k]}-C_{[j]}\right)+\sum_{J_{j} \in T} \gamma_{[j]} \\
& =(\alpha+k \theta) C_{[k]}-\theta \sum_{j=1}^{k} C_{[j]} \\
& +\beta \sum_{j=1}^{k} a_{[j]}(1+b)^{k-j}\left[(1+b)^{m}-1\right] \\
& +\beta \sum_{j=k+1}^{k+m} a_{[j]}(1+b)^{k+m-j}+\sum_{J_{j} \in T} \gamma_{[j]}
\end{aligned}
$$




$$
\begin{aligned}
& =(\alpha+k \theta) \sum_{j=1}^{k} a_{[j]}(1+b)^{k-j} \\
& +\beta \sum_{j=1}^{k} a_{[j]}(1+b)^{k-j}\left[(1+b)^{m}-1\right] \\
& -\theta \sum_{j=1}^{k} a_{[j]} \sum_{i=0}^{k-j}(1+b)^{i}+\beta \sum_{j=k+1}^{k+m} a_{[j]}(1+b)^{k+m-j} \\
& +\sum_{j=k+m+1}^{n} \gamma_{[j]} \\
& =\sum_{j=1}^{k} a_{[j]}(1+b)^{k-j}\left\{(\alpha+k \theta)+\beta\left[(1+b)^{m}-1\right]\right\} \\
& -\theta \sum_{j=1}^{k} a_{[j]} \sum_{i=0}^{k-j}(1+b)^{i}+\beta \sum_{j=k+1}^{k+m} a_{[j]}(1+b)^{k+m-j} \\
& +\sum_{j=k+m+1}^{n} \gamma_{[j]} \\
& =\sum_{j=1}^{k+m} w_{j} a_{[j]}+\sum_{j=k+m+1}^{n} \gamma_{[j]} .
\end{aligned}
$$

Corollary 6. If $m=n-k$, then

$$
F\left(d_{1}, D, \pi, n-k\right)=\sum_{j=1}^{n} w_{j} a_{[j]},
$$

where

$$
w_{j}=\left\{\begin{array}{lc}
\left\{(\alpha+k \theta)+\beta\left[(1+b)^{m}-1\right]\right\}(1+b)^{k-j} \\
-\theta \sum_{i=0}^{k-j}(1+b)^{i}, & j=1,2, \ldots, k \\
\beta(1+b)^{k+m-j}, & j=k+1, k+2, \ldots, n .
\end{array}\right.
$$

Equation (11) can be viewed as the scalar product of two vectors, $w_{j}$ and $a_{[j]}$, respectively, $(j=1, \ldots, n)$. It is well known (from Hardy et al. [22]) that (11) is minimized by sorting the elements of the $w_{j}$ and $a_{[j]}$ vectors in opposite orders. This procedure can be done in $O(n \log n)$ time. We refer to this rule as the HLP rule in the rest of the paper.

Theorem 7. If the number of $\bar{D}$ jobs is given, then the problem $1\left|P_{j}=a_{j}+b t\right| \alpha d_{1}+\beta D+\theta \sum_{J_{j} \in E} E_{j}+\sum_{J_{j} \in T} \gamma_{j}$ can be formulated as an assignment problem.

Proof. We define $z_{j r}$ as a $0 / 1$ variable such that $z_{j r}=1$ if job $J_{j}$ is scheduled in position $r$, and $z_{j r}=0$, otherwise. We can formulate the problem $1\left|P_{j}=a_{j}+b t\right| \alpha d_{1}+\beta D+$ $\theta \sum_{J_{j} \in E} E_{j}+\sum_{J_{j} \in T} \gamma_{j}$ as the following assignment problem:

$$
\operatorname{AP}(m) \quad \text { Min } \quad \sum_{j=1}^{n} \sum_{r=1}^{n} C_{j r}^{m} z_{j r}
$$

Subject to

$$
\begin{aligned}
& \sum_{r=1}^{n} z_{j r}=1, \quad j=1,2, \ldots, n \\
& \sum_{j=1}^{n} z_{j r}=1, \quad r=1,2, \ldots, n \\
& z_{j r}=0 \text { or } 1, \quad j, r=1,2, \ldots, n,
\end{aligned}
$$

where

$$
C_{j r}^{m}=\left\{\begin{array}{cc}
a_{j}\left[(\alpha+k \theta)+\beta\left((1+b)^{m}-1\right)\right)(1+b)^{k-r} \\
\left.-\theta \sum_{i=0}^{k-r}(1+b)^{i}\right], & r=1,2, \ldots, k \\
a_{j}\left[\beta(1+b)^{k+m-r}\right], & r=k+1, k+2, \ldots, k+m \\
\gamma_{j}, & r=k+m+1, \ldots, n .
\end{array}\right.
$$

Therefore, based on the above analysis, we can obtain a polynomial algorithm for the problem $1\left|P_{j}=a_{j}+b t\right|$ $\alpha d_{1}+\beta D+\theta \sum_{J_{j} \in E} E_{j}+\sum_{J_{j} \in T} \gamma_{j}$.

\section{Algorithm 8.}

Step 0. By Lemma 4, calculate $k=\lceil(\beta-\alpha) / \theta\rceil$.

Step 1. For $m$ from 0 to $n-k-1$, solve the above assignment problem $\mathrm{AP}(m)$ to obtain a local optimal schedule and the total cost $F(m)$.

Step 2. For $m=n-k$, first calculate the positional weights defined by (12) and assign the $\mathrm{n}$ jobs to the corresponding positions according to the HLP rule and then use (11) to evaluate the objective value $F(n-k)$.

Step 3. The global optimal schedule is the one with the minimum total cost given by $\min \{F(m) \mid 0 \leq m \leq n-k\}$.

Based on the above analysis, we have the following result.

Theorem 9. The scheduling problem $1\left|p_{j}=a_{j}+b t\right| \alpha d_{1}+$ $\beta D+\theta \sum_{J_{j} \in E} E_{j}+\sum_{J_{j} \in T} \gamma_{j}$ can be solved by Algorithm 8 in $\mathrm{O}\left(n^{4}\right)$ time.

Proof. For a given $m$, our problem becomes identical to the classical assignment problem and can be solved in $O\left(n^{3}\right)$ time. Since $0 \leq m \leq n-k \leq n$, the overall time requirement of Algorithm 8 is $O\left(n^{4}\right)$. 
Example 10. Consider the instance with

$$
\begin{aligned}
& n=5, \quad \alpha=2, \quad \beta=4, \quad \theta=0.5, \quad b=0.3, \\
& a_{1}=4, \quad a_{2}=3, \quad a_{3}=6, \quad a_{4}=9, \quad a_{5}=11 \text {, } \\
& \gamma_{1}=6, \quad \gamma_{2}=4, \quad \gamma_{3}=5, \quad \gamma_{4}=3, \quad \gamma_{5}=30 \text {. }
\end{aligned}
$$

Now we apply Algorithm 8 to solve Example 10.

Step 0. Calculate the index $k=\lceil(\beta-\alpha) / \theta\rceil=\lceil(4-2) / 0.5\rceil=4$.

Step 1. When $m=0$, the $C_{j r}^{0}$ values can be calculated by (14) and given below:

$$
C_{j r}^{0}=\left(\begin{array}{ccccc}
22.7780 & 19.0600 & 16.2000 & 14 & 6 \\
17.0835 & 14.2950 & 12.1500 & 10.5 & 4 \\
34.1670 & 28.5900 & 24.3000 & 21 & 5 \\
51.2505 & 42.8850 & 36.4500 & 31.5 & 3 \\
62.6395 & 52.4150 & 44.5500 & 38.5 & 30
\end{array}\right)
$$

The optimal job sequence is $\left(J_{2}, J_{1}, J_{3}, J_{5}, J_{4}\right)$.

The optimal objective value is $F(0)=101.9435$.

Step 2. When $m=1$, the $w_{j}$ values can be calculated by (12):

$$
\begin{gathered}
w_{1}=8.3309, \quad w_{2}=6.7930, \quad w_{3}=5.6100, \\
w_{4}=4.7000, \quad w_{5}=4.0000 .
\end{gathered}
$$

The optimal job sequence is $\left(J_{2}, J_{1}, J_{3}, J_{4}, J_{5}\right)$.

The optimal objective value is $F(1)=172.1247$.

Step 3. The global optimal objective is $\min \{F(0), F(1)\}=$ 101.9435. The global optimal schedule is $\left(J_{2}, J_{1}, J_{3}, J_{5}, J_{4}\right)$.

3.2. Job-Position-Dependent Learning Effect Model. By the same way as in the previous subsection, we consider the following scheduling problem: $1\left|P_{j}=a_{j} r^{a}\right| \alpha d_{1}+\beta D+$ $\theta \sum_{J_{j} \in E} E_{j}+\sum_{J_{j} \in T} \gamma_{j}$.

Lemma 11. For a given schedule $\pi=\left(J_{[1]}, J_{[2]}, \ldots, J_{[n]}\right)$, if the starting time of the first job is 0 , then $C_{[r]}=\sum_{j=1}^{r} a_{[j]} j^{a}$ and $\sum_{j=1}^{n} C_{j}=\sum_{j=1}^{n} a_{[j]}(n+1-j) j^{a}$.

Lemma 12. For the problem $1\left|p_{j}=a_{j} r^{a}\right| \alpha d_{1}+\beta D+$ $\theta \sum_{J_{j} \in E} E_{j}+\sum_{J_{j} \in T} \gamma_{j}$, if the job sequence is $\pi=\left(J_{[1]}, J_{[2]}\right.$, $\left.\ldots, J_{[n]}\right)$ and $m=|\bar{D}|$, then the objective function can be expressed as

$$
F\left(d_{1}, D, \pi, m\right)=\sum_{j=1}^{k+m} \bar{w}_{j} a_{[j]}+\sum_{j=k+m+1}^{n} \gamma_{[j]},
$$

where $\bar{w}_{j}=\left\{\begin{array}{cc}(\alpha-\theta+\theta j) j^{a}, & j=1,2, \ldots, k ; \\ \beta j^{a} & j=k+1, k+2, \ldots, k+m .\end{array}\right.$
Proof. By Lemmas 3 and 11, we have

$$
\begin{aligned}
& F(d, D, \pi, m) \\
& =\alpha d_{1}+\beta D+\theta \sum_{J_{j} \in E} E_{j}+\sum_{J_{j} \in T} \gamma_{j} \\
& =\alpha C_{[k]}+\beta \sum_{j=k+1}^{k+m} a_{[j]}(1+b)^{n-j} \\
& +\theta \sum_{j=1}^{k}\left(C_{[k]}-C_{[j]}\right)+\sum_{J_{j} \in T} \gamma_{[j]} \\
& =(\alpha+k \theta) C_{[k]}-\theta \sum_{j=1}^{k} C_{[j]}+\beta \sum_{j=k+1}^{k+m} a_{[j]}(1+b)^{n-j} \\
& +\sum_{J_{j} \in T} \gamma_{[j]} \\
& =(\alpha+k \theta) \sum_{j=1}^{k} a_{[j]} j^{a}-\theta \sum_{j=1}^{k} a_{[j]}(k+1-j) j^{a} \\
& +\beta \sum_{j=k+1}^{k+m} a_{[j]} j^{a}+\sum_{j=k+m+1}^{n} \gamma_{[j]} \\
& =\sum_{j=1}^{k+m} w_{j} a_{[j]}+\sum_{j=k+m+1}^{n} \gamma_{[j]} .
\end{aligned}
$$

Corollary 13. If $m=n-k$, then

$$
F\left(d_{1}, D, \pi, m\right)=\sum_{j=1}^{n} \bar{w}_{j} a_{[j]},
$$

where $\bar{w}_{j}=\left\{\begin{array}{cc}(\alpha-\theta+\theta j) j^{a}, & \begin{array}{c}j=1,2, \ldots, k \\ \beta j^{a}\end{array} \\ j=k+1, k+2, \ldots, k+m\end{array}\right.$

Equation (20) can be viewed as the scalar product of two vectors, $\bar{w}_{j}$ and $a_{[j]}$ vectors, respectively. The procedure can be done in $O(n \log n)$ time by the HLP rule.

Theorem 14. If we fix the number of $\bar{D}$ jobs, then the problem $1\left|p_{j}=a_{j} r^{a}\right| \alpha d_{1}+\beta D+\theta \sum_{J_{j} \in E} E_{j}+\sum_{J_{j} \in T} \gamma_{j}$ can be formulated as an assignment problem.

Proof. It is similar to the proof of Theorem 7.Again, we can define

$$
\bar{C}_{j r}^{m}= \begin{cases}(\alpha-\theta-\theta r) r^{a} a_{j}, & r=1,2, \ldots, k \\ \beta r^{a} a_{j}, & r=k+1, k+2, \ldots, k+m \\ \gamma_{j}, & r=k+m+1, \ldots, n,\end{cases}
$$

as the cost of assigning job $J_{j}(j=1,2, \ldots, n)$ to the $r$ th $(r=1,2, \ldots, n)$ position in the schedule. Then the problem 
$1\left|p_{j}=a_{j} r^{a}\right| \alpha d_{1}+\beta D+\theta \sum_{J_{j} \in E} E_{j}+\sum_{J_{j} \in T} \gamma_{j}$ can be formulated as the following assignment problem:

$$
\begin{array}{ll}
\operatorname{AP}(m) \quad \text { Min } \quad \sum_{j=1}^{n} \sum_{r=1}^{n} \bar{C}_{j r}^{m} z_{j r} \\
\text { Subject to } \\
\sum_{r=1}^{n} z_{j r}=1, \quad j=1,2, \ldots, n \\
\sum_{j=1}^{n} z_{j r}=1, \quad r=1,2, \ldots, n \\
z_{j r}=0 \text { or } 1, \quad j, r=1,2, \ldots, n .
\end{array}
$$

Similar to Section 3.1, we have the following theorem.

Theorem 15. The scheduling problem $1\left|p_{j}=a_{j} r^{a}\right| \alpha d_{1}+$ $\beta D+\theta \sum_{J_{j} \in E} E_{j}+\sum_{J_{j} \in T} \gamma_{j}$ can be solved in $O\left(n^{4}\right)$ time.

\section{Conclusions}

We have considered the single machine due-window assignment scheduling problem with variable job processing times. The objective is to minimize a linear combination of earliness, tardiness, the window location, window size, and weighted number of tardy jobs. We proposed a polynomialtime algorithm, respectively, for the learning effect and the deteriorating jobs. Obviously, if $a>0$ (i.e., deterioration or aging effect) and $b<0$ (i.e., shortening processing times), then the results of this paper still hold. In future research, we plan to explore more realistic settings, such as group technology scheduling problems, flexible flow shop scheduling problems, and unrelated parallel machines scheduling problems, or optimize other performance measures with variable job processing time.

\section{Conflict of Interests}

Yu-Bin $\mathrm{Wu}$ and Ping Ji declare that there is no conflict of interests regarding the publication of this paper.

\section{Acknowledgments}

The authors are grateful to the anonymous referees for their helpful comments on earlier versions of this paper. This research was supported by the Science Research Foundation of Shenyang Aerospace University (Grant no. 201304Y) and the Research Grants Council of the Hong Kong Special Administrative Region, China (Project no. PolyU 517011).

\section{References}

[1] D. Biskup, "A state-of-the-art review on scheduling with learning effects," European Journal of Operational Research, vol. 188, no. 2, pp. 315-329, 2008.
[2] S. Gawiejnowicz, Time-Dependent Scheduling, Springer, Berlin, Germany, 2008.

[3] R. Rudek, "Scheduling problems with position dependent job processing times: computational complexity results," Annals of Operations Research, vol. 196, pp. 491-516, 2012.

[4] J.-B. Wang and M.-Z. Wang, "Worst-case behavior of simple sequencing rules in flow shop scheduling with general positiondependent learning effects," Annals of Operations Research, vol. 191, pp. 155-169, 2011.

[5] L.-H. Sun, K. Cui, J.-H. Chen, J. Wang, and X.-C. He, "Research on permutation flow shop scheduling problems with general position-dependent learning effects," Annals of Operations Research, vol. 211, pp. 473-480, 2013.

[6] L.-H. Sun, K. Cui, J.-H. Chen, J. Wang, and X.-C. He, "Some results of the worst-case analysis for flow shop scheduling with a learning effect," Annals of Operations Research, vol. 211, pp. 481490, 2013.

[7] Y. Y. Lu, G. Li, Y. B. Wu, and P. Ji, “Optimal due-date assignment problem with learning effect and resource-dependent processing times," Optimization Letters, vol. 8, no. 1, pp. 113-127, 2014.

[8] J.-B. Wang and M.-Z. Wang, "Single-machine due-window assignment and scheduling with learning effect and resourcedependent processing times," Asia-Pacific Journal of Operational Research, vol. 31, no. 5, Article ID 1450036, 2014.

[9] J.-B. Wang and M.-Z. Wang, "Single-machine scheduling with nonlinear deterioration," Optimization Letters, vol. 6, no. 1, pp. 87-98, 2012.

[10] J.-B. Wang and M.-Z. Wang, "Minimizing makespan in threemachine flow shops with deteriorating jobs," Computers and Operations Research, vol. 40, no. 2, pp. 547-557, 2013.

[11] X.-R. Wang and J.-J. Wang, "Single-machine scheduling with convex resource dependent processing times and deteriorating jobs," Applied Mathematical Modelling, vol. 37, no. 4, pp. 23882393, 2013.

[12] Y.-T. Xu, Y. Zhang, and X. Huang, "Single-machine ready times scheduling with group technology and proportional linear deterioration," Applied Mathematical Modelling, vol. 38, no. 1, pp. 384-391, 2014.

[13] T. C. E. Cheng, S.-J. Yang, and D.-L. Yang, "Common duewindow assignment and scheduling of linear time-dependent deteriorating jobs and a deteriorating maintenance activity," International Journal of Production Economics, vol. 135, no. 1, pp. 154-161, 2012.

[14] S.-J. Yang, H.-T. Lee, and J.-Y. Guo, "Multiple common due dates assignment and scheduling problems with resource allocation and general position-dependent deterioration effect," International Journal of Advanced Manufacturing Technology, vol. 67, no. 1-4, pp. 181-188, 2013.

[15] J. Liu, Y. Wang, and X. Min, "Single-machine scheduling with common due-window assignment for deteriorating jobs," Journal of the Operational Research Society, vol. 65, no. 2, pp. 291-301, 2014.

[16] J.-B. Wang and C. Wang, "Single-machine due-window assignment problem with learning effect and deteriorating jobs," Applied Mathematical Modelling, vol. 35, no. 8, pp. 4017-4022, 2011.

[17] J.-B. Wang, L. Liu, and C. Wang, "Single machine SLK/DIF due window assignment problem with learning effect and deteriorating jobs," Applied Mathematical Modelling, vol. 37, no. 18-19, pp. 8394-8400, 2013. 
[18] S. Li, C. T. Ng, and J. Yuan, "Scheduling deteriorating jobs with CON/SLK due date assignment on a single machine," International Journal of Production Economics, vol. 131, no. 2, pp. 747-751, 2011.

[19] Y. Yin, T. C. E. Cheng, C.-J. Hsu, and C.-C. Wu, "Singlemachine batch delivery scheduling with an assignable common due window," Omega, vol. 41, no. 2, pp. 216-225, 2013.

[20] D. Biskup, "Single-machine scheduling with learning considerations," European Journal of Operational Research, vol. 115, no. 1, pp. 173-178, 1999.

[21] R. L. Graham, E. L. Lawler, J. K. Lenstra, and A. H. R. Kan, "Optimization and approximation in deterministic sequencing and scheduling: a survey," Annals of Discrete Mathematics, vol. 5, pp. 287-326, 1979.

[22] G. H. Hardy, J. E. Littlewood, and G. Polya, Inequalities, Cambridge University Press, Cambridge, UK, 1967. 


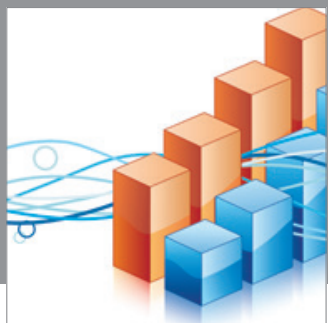

Advances in

Operations Research

mansans



The Scientific World Journal
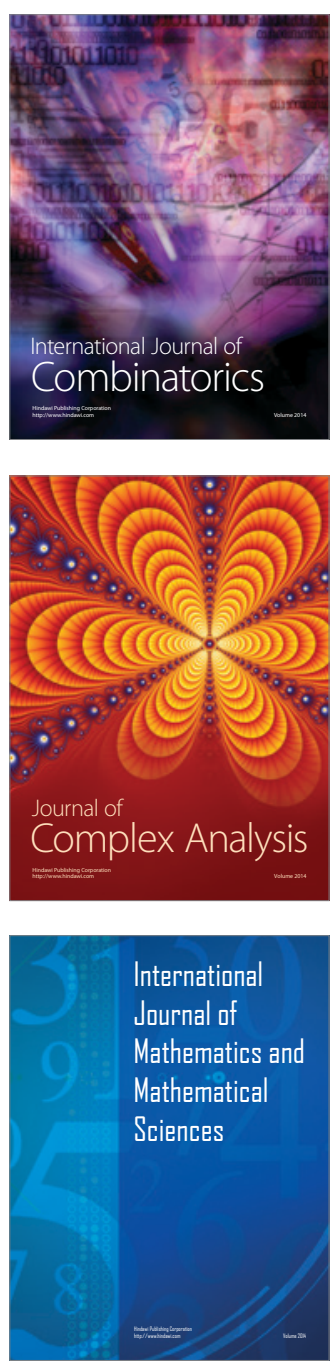
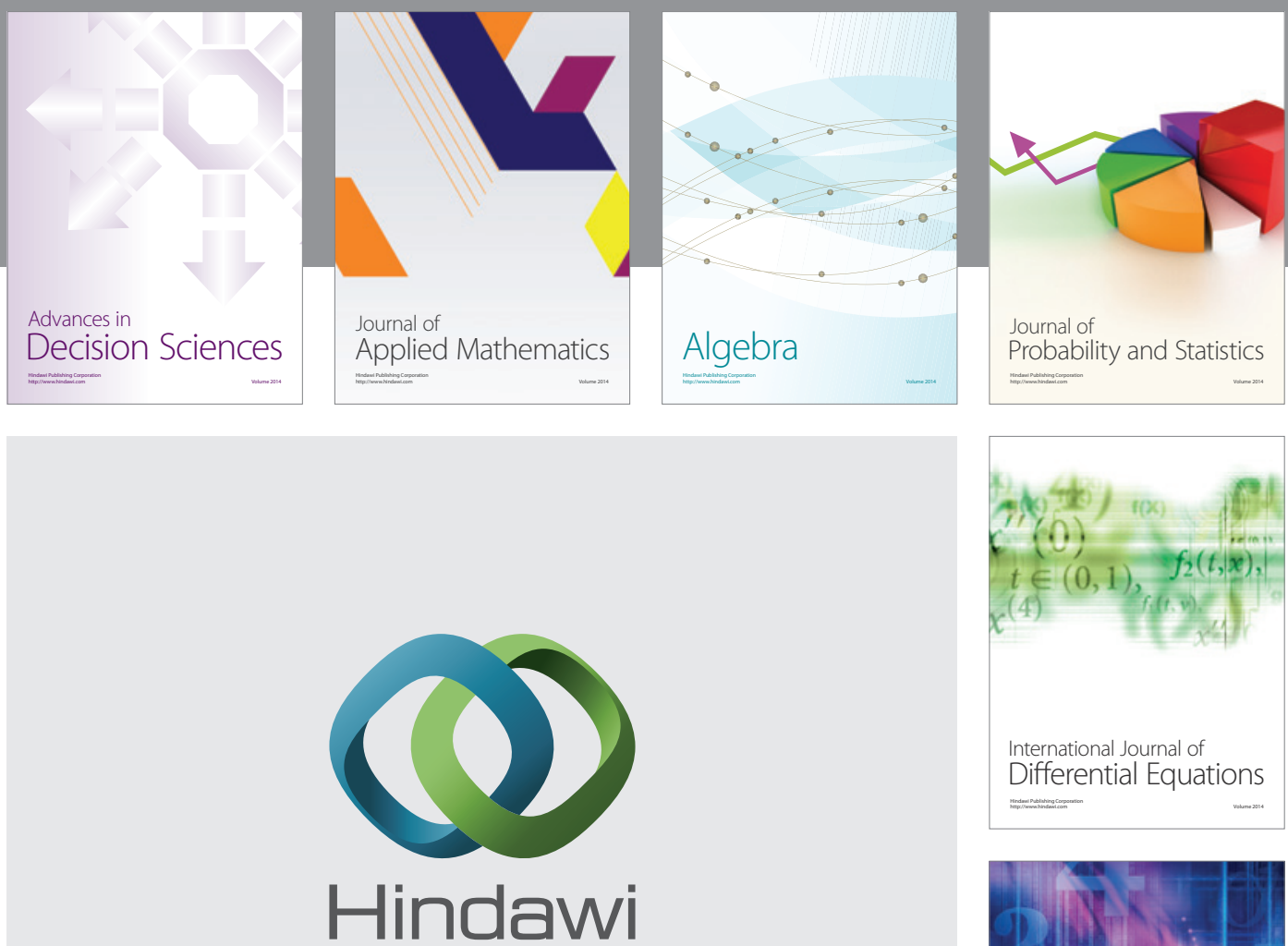

Submit your manuscripts at http://www.hindawi.com
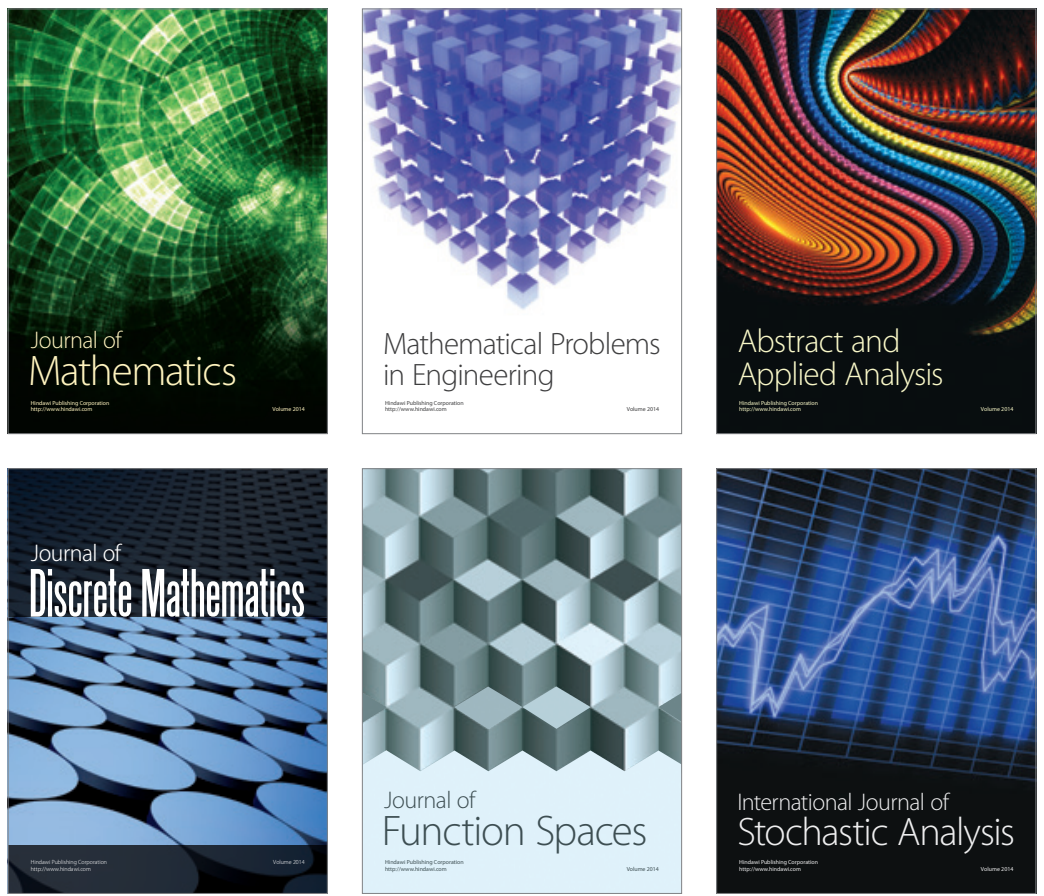

Journal of

Function Spaces

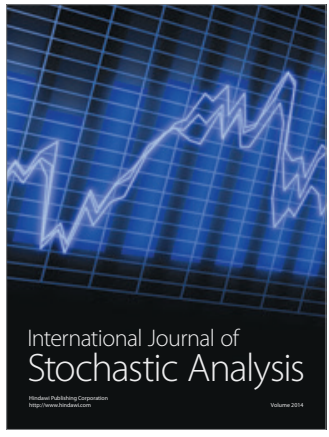

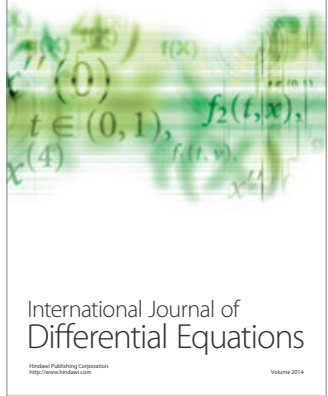
